\title{
TWENTY MINUTES' TALK ON GONOCOCCAL CONJUNCTIVITIS
}

\author{
By ADRIAN CADDY, M.D., F.R.C.S., Surgeon Royal Westminster \\ Ophthalmic Hospital
}

Mr. President, Ladies and Gentlemen,-This subject does not lend itself so readily as the last to illustration by slides, but I shall ask you afterwards to see slides and specimens in the laboratory.

There are three types of people who have gonococcal conjunctivitis: the new-born baby, the adult who is the subject of gonorrhœa, and the person who is suffering from trachoma, in whom the conjunctivitis is an added infection. Trachomia is not often seen in England but in tropical countries, especially Egypt and India; I saw much of it in India. Every now and then one found that an acute infection would be grafted on to trachoma, and a large proportion of the cases are due to gonococcal conjunctivitis. This inflammation does not mean that the patient has gonorrhœa, he has no venereal infection. The trouble is often epidemic in character. In the majority of cases it is gonococcal, but there is often present a streptococcus, or there may be any organism which causes acute conjunctivitis.

Acute conjunctivitis is often curative of trachoma. Twenty years ago we injected into the conjunctiva for trachoma an extract of jequirity, which caused an acute conjunctivitis and so tended to cure the trachoma.

New-born babies get the disease from infection of the genital passages of the mother, and occasionally babies are actually born with it, this fact showing that it may be an inter-uterine infection. This has been proved by finding it in cases after Cæsarean section, gonococcal conjunctivitis developing before the birth of the baby. We know that the infection could not have developed earlier than two months before the birth of the child, because its eyelids are sealed together two and a half months before the child is born.

Ophthalmia neonatorum is responsible for 50 per cent. 


\section{TALK ON GONOCOCCAL CONJUNCTIVITIS}

of the blind children, and 8 per cent. of the total number of the blind. The reason that it is so dangerous is that the gonococcus can attack the healthy conjunctiva and cornea without there being any precedent corneal lesion. Many infections need a prior breach of surface, but that is not necessary with the gonococcus. The corneal ulceration, which is virulent, goes on to perforation of the eye, and there is destruction of the lens, iris, etc., and they are extruded.

What happens in a small child is that it develops ophthalmia neonatorum and gets a corneal ulcer. This forms a scar over the cornea, and so hinders light getting into the eye. It is essential that light should get into the eye in the first six weeks of extra-uterine life, for if it is prevented from getting into the eye it means that the retina does not cievelop, the child has no power of fixation, and it develops nystagmus, and the vision is very defective. So it is very important that, in the case of the child, there shall be no interference with the path of light into the retina. Of purulent conjunctivitis in babies only 60 per cent. to 70 per cent. are gonorrhœal the remaining 30 per cent. to 40 per cent. being due to streptococci or other organisms.

The incubation period is from a few hours to two or three days, and nowadays, it is very rare; I doubt if our registrar has been able to produce a case of ophthalmia neonatorum to show you this afternoon. Since the recent careful treatment by midwives of infants' eyes the only sequel is that the eyes are sometimes filled up so vigorously with silver nitrate that a chemical irritation ensues, leading to infection with pneumococci, and this you may find develop a few weeks after the child is born. It is not due to the gonococcus, but to the drastic treatment.

The infection of the eye is much more severe in adults; so much so that it often results in destruction of the eye. But fortunately it is rare in adults; we find that about I in 700 cases of gonorrhœa develops gonococcal conjunctivitis.

Why are these cases so liable to perforation and damage? Because the conjunctiva swells up and the margins of the cornea are bathed in pus. Ulceration spreads from that, and perforation speedily occurs. In the baby it is usually bilateral ; if it affects only one eye 


\section{BRITISH JOURNAL OF VENEREAL DISEASES}

in a baby it is not likely to be gonococcal, but due to lachrimal obstruction, the lachrimal passage being blocked up with epithelial débris. Frequently these latter cases get infected with pneumococci.

Now as to the treatment of a case of conjunctivitis. First there is preventive treatment. On the birth of the child it is washed and I per cent. silver nitrate solution is dropped into the eye. There is Meibomian secretion gluing the lids together, and there is not much tendency for anything to be pushed into the eye, except by forceps. If the patient is already infected with the trouble, what treatment will you adopt? Constantly wash the eye out with I in I0,000 perchloride of mercury; do this steadily every two hours. What is beneficial is not so much the antiseptic as the mechanical effect of the water. Boric acid can be used for the purpose and is probably equally efficient. And once a day the lids should be everted, if possible, and the eye painted out with a 2 per cent. solution of silver nitrate. This has a specially valuable effect on the eye because it produces a superficial slough of the surface of the conjunctiva, in which three-fourths of the organisms are present. In later stages we use argyrol and protargol. Similar in their action but weaker and less painful.

For adults do the same thing. In the early days, before there is a free secretion of pus, you rely on the washing alone; when the secretion is free, use silver nitrate.

Do not tie up the eyes, you want the discharge to escape. Put a little zinc or boric ointment round the eyelids, to prevent soreness due to washing out.

For babies, vaccine treatment is useless, but it is excellent for adults, and Dr. Browning, bacteriologist at Moorfields, favours large doses of vaccines.

In an adult affected with gonococcal conjunctivitis in one eye you must protect the other eye, by using a watchglass or sticking-plaster, so that there will be no risk of the infection being carried to the fellow eye.

The disease lasts weeks. If there are signs of corneal ulcer you must use atropine, as that will allay inflammation and will obviate the risk of perforation of the eye. If the cornea is invaded, an ulcer forms, this bursts, and the whole contents of the eye come out. This is known as phthisis bulbi. If you have a case of phthisis 


\section{TALK ON GONOCOCCAL CONJUNCTIVITIS}

bulbi in a small child, the socket will shrink, and a glass eye cannot be inserted. Moreover, the face does not grow normally, and that side of the face differs from the other, and the child is lop-sided. So be cautious about removing a child's eye if it can be avoided.

Specimens Demonstrated after the Address in the Patholcgical Laboratory of THE Royal WestMinster OPHThalmic Hospital

(i.) Section of a normal eye. Incidentally this eye contains a well-marked sarcome of the choroid in one corner but the rest of the eye is perfectly healthy.

(ii.) Phthisis bulbi after gonococcal conjunctivitis.

(a) museum specimen of half the eye in formalin.

(b) Celloidin block containing the other half of the eye prior to microscopic section.

(c) microscopic section of the eye.

(iii.) Staphyloma of the eye. Gonococcal conjunctivitis. This leads to corneal ulcer. The floor of the ulcer bulges under the influence of the normal intraocular pressure. The eye may get so big that the lids cannot shut over it. The conjunctiva may get corneified and even grow a horn. Three bottle specimens and two slides demonstrate these changes.

(iv.) Gonococcal conjunctivitis has led to a hypopyon ulcer of the cornea. The slide shows this ulcer.

(v.) The same thing only more advanced causing hernia of the iris and lens.

(vi.) Slides showing (i.) gonococci from a child's eye.

(ii.) streptococci from another eye

(iii.) Bacillus of Morax and Axenfeld. A big bacillus which causes angular conjunctivitis. 